\title{
Managing Performance and Efficiency of a Processor
}

\author{
Aditi Shinde and Vishwani D. Agrawal \\ Department of Electrical and Computer Engineering \\ Auburn University, Auburn, AL 36849 \\ Email: ajs0042@tigermail.auburn.edu,vagrawal@eng.auburn.edu
}

\begin{abstract}
The performance of a processor generally means how fast it can execute a task. For a given architecture we can measure the size of a task as the number of clock cycles it will take to execute. Then clock frequency $(f)$ will determine the execution time. Normally, the frequency can be raised if the supply voltage $V_{d d}$ is increased. This, however, increases the power and energy used. We introduce a new measure, cycle efficiency $(\eta)$ as cycles per joule that gives the rate of computational work per unit energy. Similar to $f, \eta$ is also a function of $V_{d d}$. We provide a method of characterizing a processor in terms of its $f$ and $\eta$ versus $V_{d d}$ characteristics. Intel Pentium $M$ processor with an assumed 90nm CMOS PTM (predictive technology model) is used as an example. For a demonstration of performance and energy management, we consider a program that executes in 1.8 billion clock cycles. At the nominal operating supply of $1.2 \mathrm{~V}$ we have $f=1.8 \mathrm{GHz}$ and $\eta=15$ megacycles/joule. The program executes in 1 second and uses 120 joules. For operation at $0.6 \mathrm{~V}, f=277 \mathrm{MHz}$ and $\eta=70$ megacycles/joule, resulting in a run time of 6.5 seconds and consumption of 25 joules. We also find a subthreshold voltage extreme of $200 \mathrm{mV}$, $f=54.5 \mathrm{MHz}$ and $\eta=660$ megacycles/joule. Now the program will take 33 seconds but will consume only 2.27 joules. Thus, using cycle efficiency and clock frequency one can manage the time and energy performances according to the requirements of a computing task.
\end{abstract}

\section{INTRODUCTION}

Voltage scaling has been a very popular low power design methodology in the industry. Having a quadratic relationship with the power consumed, lowering the supply voltage reduces the power considerably. But this mars the overall performance of the circuit. Optimizing performance and power simultaneously requires a thorough study of the available resources and trade-offs possible. This paper looks into one of the most important areas of contemporary research in electrical and computer engineering: energy efficiency [4], [6], [16], [17]. Power and Performance are two conflicting goals a designer has to achieve [11]-[13], [15]. With a number of performance oriented devices emerging with a huge demand of power from a fixed capacity battery, using the battery wisely becomes important [9], [10]. This paper suggests a new metric called cycle efficiency that can be considered while deciding upon the operating conditions of a processor for energy efficiency.

\section{CyCle EFFICIENCY}

Performance of a processor refers to its performance in time. It is defined for a task (or program) as the inverse of the execution time [14]. Similarly, efficiency of a processor is defined [14] as the inverse of the energy consumed by the program. Thus,

$$
\begin{aligned}
\text { Performance } & =\frac{1}{\text { Execution time }} \\
\text { Efficiency } & =\frac{\text { Performance }}{\text { Power dissipation }} \\
& =\frac{1}{\text { Energy consumed }}
\end{aligned}
$$

We observe a similarity between the two measures. The performance can be called time efficiency and efficiency can be referred to as energy performance. In this paper, we will call them time performance and energy performance, respectively. If we regard the clock cycle as a unit of work that a processor performs, then a clock cycle means a time period $1 / f$, where $f$ is the frequency in units of cycles per second or hertz $(\mathrm{Hz})$. A clock cycle also means certain amount of energy or energy per cycle $(E P C)$. We define cycle efficiency, $\eta=1 / E P C$, its unit being cycles per joule. Thus, a clock cycle means $1 / f$ second in time and $1 / \eta$ joule in energy.

Consider a program being run on a processor. Suppose the program execution takes $C$ clock cycles. Then we have,

$$
\text { Execution time }=\frac{C}{f}
$$

and

$$
\text { Energy consumed }=\frac{C}{\eta}
$$

where $\eta$ is cycle efficiency of the processor in cycles per joule. 
Equation 1 gives the time performance of the processor as,

$$
\text { Performance in time }=\frac{1}{\text { Execution time }}=\frac{f}{C}
$$

Similarly, Equation 2 gives the energy performance as,

$$
\text { Performance in energy }=\frac{1}{\text { Energy consumed }}=\frac{\eta}{C}
$$

Clearly, cycle efficiency $(\eta)$ characterizes the energy performance in a similar way as frequency $(f)$ characterizes the time performance. These two performance parameters are related to each other by the power being consumed, as follows:

$$
\text { Power }=\frac{f}{\eta}
$$

\section{ENERGy AND DELAY FOR A TECHNOLOGY}

We assume that the processor being characterized is large and a full scale gate level or transistor level model may not be available. Even if such a model was available, a detailed simulation for various voltages would be impractical for high complexity reason. However, operational data about the processor, such as voltage, maximum clock frequency and power consumption, is available. Also, the technology of the device is specified. We, therefore, characterize the technology using known and easily analyzable circuits. Then, we scale the characterization to the processor.

For a given technology, we derive energy consumption and delay as functions of supply voltage. The procedure given here can be used for any technology for which simulation models are available. For illustration, we use 90nm CMOS PTM technology [20] and the Hspice simulator [1].

Energy per Cycle (EPC). Figure 1 shows the energy per cycle (EPC) for an eight-bit ripple carry adder circuit. This result is available from recent references [5], [8]. The circuit was synthesized using a 90nm PTM [20] CMOS technology library and simulated with random input vectors. The vector period was set close to the critical path delay, which was determined by simulation of a vector pairs generated to activate the critical path. Both dynamic $\left(E_{d y n}\right)$ and leakage $\left(E_{\text {leak }}\right)$ energy components were determined using Hspice [1]. Simulation was repeated for voltage range $80 \mathrm{mV}$ to $1.2 \mathrm{~V}$. EPC in Figure 1 is $E_{t o t}=E_{d y n}+E_{\text {leak }}$ and is shown by solid line. Threshold voltages are $0.29 \mathrm{~V}$ for nMOS and $-0.21 \mathrm{~V}$ for pMOS devices. We notice that EPC is minimum at $0.17 \mathrm{~V}$, which a sub-threshold voltage. At this voltage the leakage and dynamic energies are equal, the circuit operates extremely slow but is most energy efficient [2], [5], [18].

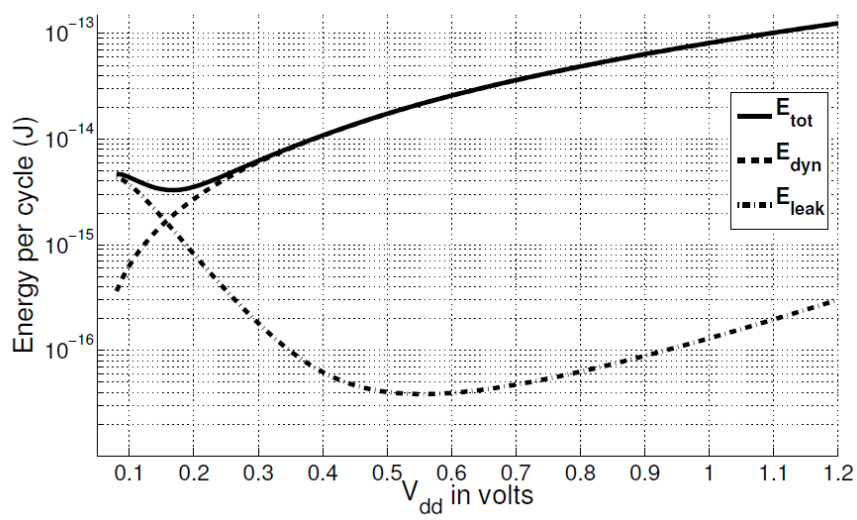

Fig. 1. Hspice [1] characterization of 8-bit ripple-carry adder in 90nm PTM technology [5], [7], [8].

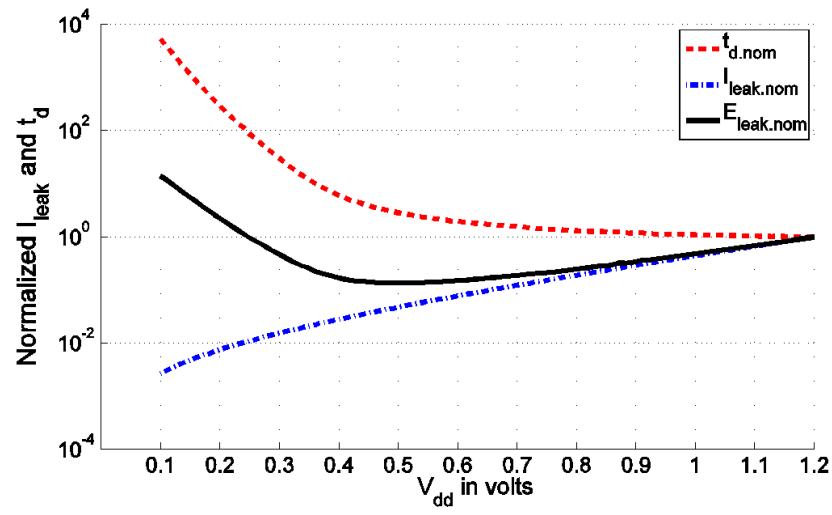

Fig. 2. delay and leakage current normalized to an inverter at $V_{D D}=1.2 \mathrm{~V}$ through Hspice simulation in PTM 90nm CMOS [5], [7], [8].

Delay. For delay we used a recent result for a chain of $90 \mathrm{~nm}$ CMOS inverters [5], [8]. This is shown in Figure 2 where the delay is normalized with respect to the delay at $1.2 \mathrm{~V}$. We note that as the supply voltage drops below $1.2 \mathrm{~V}$, the delay $\left(t_{d}\right)$ increases gradually at first and then rather rapidly as we approach the subthreshold range.

\section{Characterization of Intel Pentium M PROCESSOR}

We characterize the Intel Pentium M processor in the $90 \mathrm{~nm}$ CMOS technology. The exact details of its technology are not known to us, so for illustrative purpose we assume the CMOS predictive technology model (PTM) [20] as analyzed in the previous section.

Processor data is obtained from a published paper [4]. For a supply voltage $V_{d d}=1.2 \mathrm{~V}$, the maximum clock rate is $1.8 \mathrm{GHz}$ and the chip consumes $120 \mathrm{~W}$. This gives an energy per cycle, $E P C=120 /\left(1.8 \times 10^{9}\right)=66.67 \mathrm{~nJ}$. Scaling the graph of 


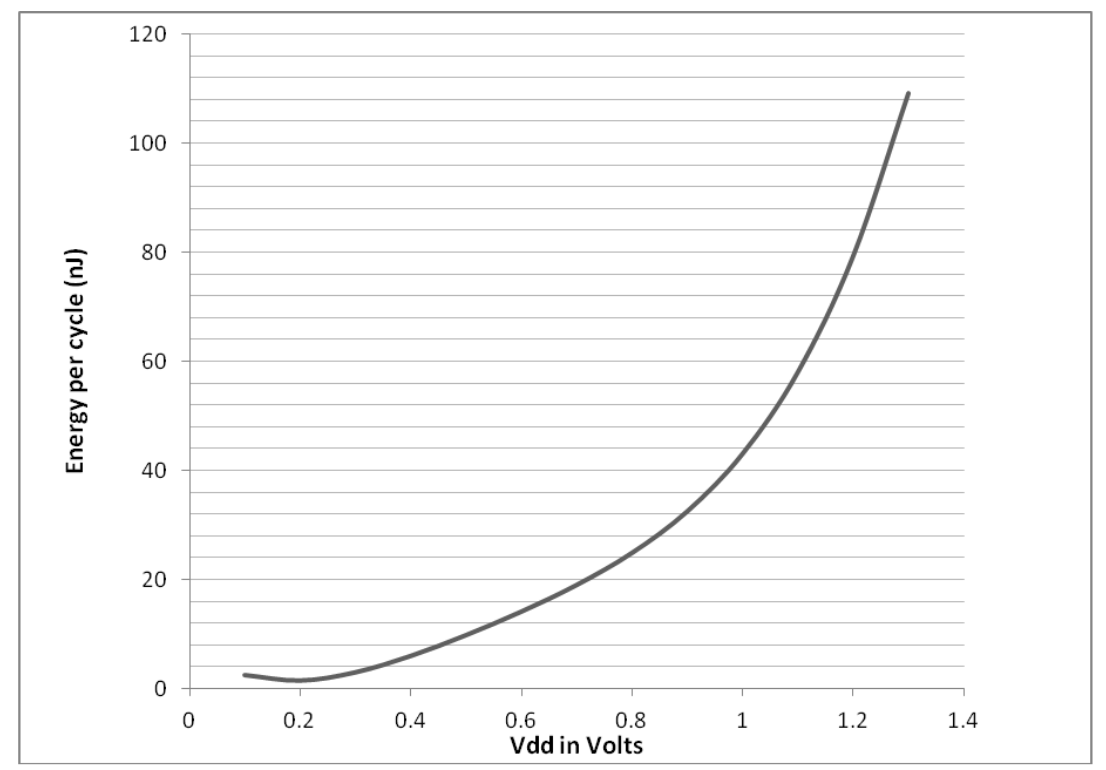

Fig. 3. Energy per cycle for Intel Pentium M Processor in 90nm CMOS PTM [20].

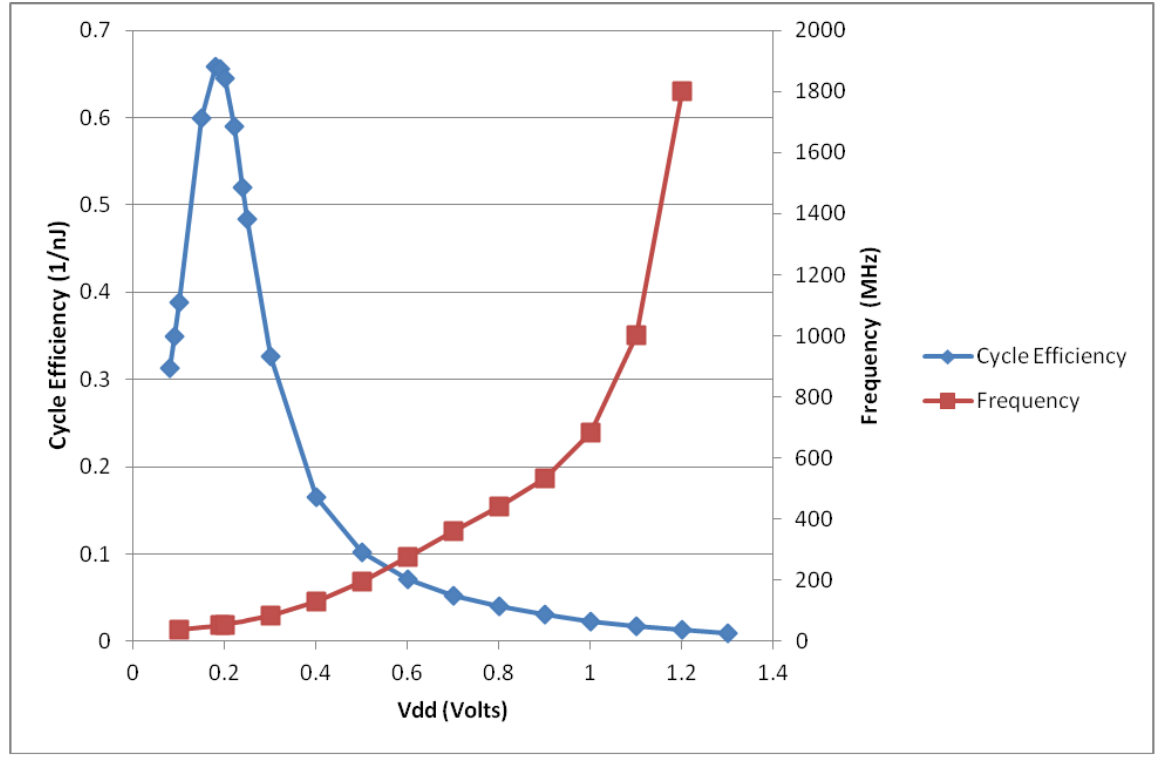

Fig. 4. Cycle efficiency $(\eta)$ and frequency $(f)$ versus supply voltage $\left(V_{d d}\right)$ for Pentium M processor in 90nm CMOS PTM [20].

Figure 1 to read an EPC of $66.67 \mathrm{~nJ}$ at $V_{d d}=1.2 \mathrm{~V}$ we get the EPC versus $V_{d d}$ characteristic for Pentium M processor as shown in Figure 3. The inverse of $E P C$ is the cycle efficiency $\eta$, which is shown in Figure 4.

Next, we scale the delay $t_{d}$ versus $V_{d d}$ characteristic of Figure 2 for Pentium $M$ processor so that the frequency at $V_{d d}=1.2 \mathrm{~V}$ is $1.8 \mathrm{GHz}$. The scaled frequency $(f)$ versus $V_{d d}$ characteristic is also shown in Figure 4.

\section{Managing Processor Power}

Consider a program that executes in 1.8 billion clock cycles. Three scenarios are given in Table I. If we operate the processor at $1.2 \mathrm{~V}$, we see From Figure 4 that clock frequency $f=1.8 \mathrm{GHz}$ and cycle efficiency $\eta=15$ megacycles/joule. The program is executed in one second, the processor consumes $120 \mathrm{~W}$ of power and the total energy used is 120 joules. If we reduce the supply voltage to half, i.e., $V_{d d}=0.6 \mathrm{~V}$, and run the processor at $f=277 \mathrm{MHz}$ as given by Figure 4 , 
TABLE I

TIME AND ENERGY FOR A PROGRAM THAT EXECUTES IN 1.8 BILLION CLOCK CYCLES FOR VARIOUS OPERATING VOLTAGES AND CLOCK FREQUENCIES.

\begin{tabular}{|c|c|c|c|c|c|}
\hline $\begin{array}{c}\text { Voltage } V_{d d} \\
\text { volts }\end{array}$ & $\begin{array}{c}\text { Clock frequency } f \\
\mathrm{MHz}\end{array}$ & $\begin{array}{c}\text { Cycle efficiency } \eta \\
\text { megacycles/joule }\end{array}$ & $\begin{array}{c}\text { Power }=\frac{f}{\eta} \\
\text { watts }\end{array}$ & $\begin{array}{c}\text { Execution time } \\
\text { seconds }\end{array}$ & $\begin{array}{c}\text { Total energy consumed by program } \\
\text { joules }\end{array}$ \\
\hline \hline 1.2 & 1800 & 15 & 120 & 1.0 & 120 \\
0.6 & 277 & 70 & 39.6 & 6.5 & 25 \\
0.2 & 54.5 & 660 & 0.083 & 33 & 2.72 \\
\hline
\end{tabular}

which also gives cycle efficiency $\eta=70$ megacycles/joule. Now the power consumption is $3.96 \mathrm{~W}$, but the program takes 6.5 seconds and uses 25 joules of energy.

The third case uses a subthreshold voltage $V_{d d}=200 \mathrm{mV}$. From Figure $4, f=54.5 \mathrm{MHz}$ and $\eta=660$ megacycles/joule. The processor runs at a very low power level of $83 \mathrm{~mW}$, but the program takes 33 seconds and uses 2.72 joules of energy. This type of slow but highly energy efficient operation has been reported by several authors [5], [18], [19].

\section{CONCLUSION}

We have introduced a new parameter, cycle efficiency $(\eta)$, expressed as cycles per joule that is similar to the clock frequency $f$ expressed as cycles per second. For a computing task, $f$ is the rate of execution in time and $\eta$ is the rate of execution in energy. To consider the analogy of automobiles, $f$ is analogous to speed in miles per hour (MPH) and $\eta$ is analogous to miles per gallon (MPG). We demonstrate how the operation of a processor can be characterized for $f$ and $\eta$ as functions of the supply voltage $V_{d d}$. Once we set $V_{d d}$ based on the time and energy requirements, we let the processor run at the fastest clock frequency allowed by the structure (critical path delay). For modern technologies that have significant leakage, this is an efficient mode of operation [3]. Given that the underlying objective is to weigh the total energy cost against the execution time, the time and energy performances can be managed using these two parameters.

\section{REFERENCES}

[1] "HSPICE User Guide: Simulation and Analysis." Synopsys Inc., www.synopsys.com.

[2] R. Dreslinski, M. Wiekowski, D. Blaauw, D. Slyvester, and T. Mudge, "Near-Threshold Computing: Reclaiming Moore's Law Through Energy Efficient Integrated Circuits," Proceedings of the IEEE, vol. 98, no. 2, pp. 253-266, Feb. 2010.

[3] R. Gonzalez, B. M. Gordon, and M. A. Horowitz, "Supply and Threshold Voltage Scaling for Low Power CMOS," IEEE Journal of Solid-State Circuits, vol. 32, no. 8, pp. 1210-1216, Aug. 1997.

[4] H. Hanson, K. Rajamani, S. Keckler, F. Rawson, S. Ghiasi, and J. Rubio, "Thermal Response to DVFS: Analysis with an Intel Pentium M," in Proc. International Symposium on Low Power Electronics and Design, Aug. 2007, pp. 219-224.

[5] K. Kim, Ultra Low Power CMOS Design. PhD thesis, Auburn University, Auburn, Alabama, May 2011.
[6] K. Kim and V. D. Agrawal, "Dual Voltage Design for Minimum Energy using Gate Slack," in Proc. International Conf. on Industrial Technology, 2011, pp. 419-424.

[7] K. Kim and V. D. Agrawal, "Minimum Energy CMOS Design with Dual Subthreshold Supply and Multiple Logic-Level Gates," in Proc. International Symp. Quality Electronic Design, 2011, pp. 689-694.

[8] K. Kim and V. D. Agrawal, "Ultra Low Energy CMOS Logic Using Below-Threshold Dual-Voltage Supply," Journal of Low Power Electronics, vol. 7, no. 4, pp. 460-470, Dec. 2011.

[9] M. Kulkarni, "Energy Source Lifetime Optimization for a Digital System through Power Management," Master's thesis, Auburn University, Auburn, Alabama, Dec. 2010.

[10] M. Kulkarni and V. D. Agrawal, "Energy Source Lifetime Optimization for a Digital System through Power Management," in Proc. 43rd IEEE Southeastern Symposium on System Theory, Mar. 2011, pp. 75-80.

[11] D. J. Lilja, Measuring Computer Performance: A Practitioner's Guide. New York: Cambridge University Press, 2000.

[12] A. J. Martin, M. Nystrom, and P. L. Penzes, ET2: A Metric for Time and Energy Efficiency of Computation, pp. 293-315. Springer, 2002.

[13] V. G. Oklobdzija, V. M. Stojanovic, D. M. Markovic, and N. Nedovic, Digital System Clocking: High Performance and Low-Power Aspects. Hoboken, NJ: Wiley-Interscience, 2005.

[14] D. A. Patterson and J. L. Hennessy, Computer Organization \& Design, the Hardware/Software Interface. San Francisco, California: Morgan Kaufman, fourth edition, 2008.

[15] V. Tiwari, R. Donnely, S. Malik, and R. Gonzalez, "Dynamic Power Management for Microprocessors: A Case Study," in Proc. 10th International Conf. VLSI Design, Jan. 1997, pp. 185192.

[16] M. Venkatasubramanian, "Energy Efficiency and Process Variation Tolerance of $45 \mathrm{~nm}$ Bulk and High-k CMOS Devices," Master's thesis, Auburn University, Auburn, Alabama, May 2011.

[17] M. Venkatasubramanian and V. D. Agrawal, "Subthreshold Voltage High-k CMOS Devices Have Lowest Energy and High Process Tolerance," in Proc. 43rd IEEE Southeastern Symp. System Theory, 2011, pp. 98-103.

[18] A. Wang, B. H. Calhoun, and A. P. Chandrakasan, SubThreshold Design for Ultra Low-Power Systems. New York: Springer, 2006.

[19] B. Zhai, R. Dreslinski, T. Mudge, D. Blaauw, and D. Sylvester, "Energy Efficient Near-Threshold Chip Multi-Processing," in Proc. IEEE International Symposium on Low-Power Electronics and Design, 2007, pp. 32-37.

[20] W. Zhao and Y. Cao, "New Generation of Predictive Technology Model for Sub-45nm Early Design Exploration," IEEE Transactions on Electron Devices, vol. 53, no. 11, pp. 2816-2823, 2006. 